rates and its associated factors among FSW in Guangdong, China.

Methods A cross-sectional study was conducted in seven cities in Guangdong province. We collected data on socio-demographic characteristics, gonorrhea and chlamydia testing uptake and health care services utilization from participants through face-to-face interview in 2017. We reported the outcomes adjusted for age, ethnic, education level, marital status, monthly income and local working time through multivariable logistic regressions.

Results Overall, 1207 people participated in the survey. The mean age of FSW was $30.69 \pm 6.75$ years. The average number of customers per week was $8.18 \pm 5.43$. Ninety $(7.46 \%)$ of them had gonorrhea testing and 125 (10.36\%) people had chlamydia testing in the last 12 months. Multivariable analysis indicated that FSW who reported having abnormal vaginal discharge $(\mathrm{aOR}=3.33,95 \% \mathrm{CI}: 2.03-5.46)$ or having ulcers in the genital area $(\mathrm{aOR}=6.16,95 \% \mathrm{CI}: 1.99-19.06)$ in the past year were more likely to have gonorrhea testing. Those who had stable partners $(\mathrm{aOR}=2.59,95 \% \mathrm{CI}: 135-4.99)$, had anal sex $\quad(\mathrm{aOR}=2.23,95 \% \mathrm{CI}:$ 1.76-2.84), had HIV testing $(\mathrm{aOR}=3.94,95 \% \mathrm{CI}: 2.34-6.65)$ and syphilis testing $(\mathrm{aOR}=3.27,95 \% \mathrm{CI}: 1.96-5.46)$ were also more likely to have gonorrhea testing. Similar as gonorrhea testing, participants who had abnormal vaginal discharge in the past year $(\mathrm{aOR}=4.09,95 \% \mathrm{CI}: 2.62-6.40)$, had ulcers in the genital $(\mathrm{aOR}=10.37, \quad 95 \% \mathrm{CI}: \quad 3.23-33.31)$, had anal sex $(\mathrm{aOR}=2.36,95 \% \mathrm{CI}: 1.54-3.60), \quad$ had HIV testing $(\mathrm{aOR}=5.16,95 \% \mathrm{CI}: \quad 3.21-8.30)$ and syphilis testing $(\mathrm{aOR}=6.90,95 \% \mathrm{CI}: 4.21-11.32)$ were more likely to have chlamydia testing.

Conclusion Gonorrhea and chlamydia testing rates remain low among FSW in China. Interventions that can further improve the uptake of gonorrhea and chlamydia are further needed. Providing gonorrhea and chlamydia testing at syphilis/HIV testing sites and among asymptomatic FSW may increase testing rate.

Disclosure No significant relationships.

\section{P707 UNDERSTANDING THE CORRELATES OF STI-HIV CO- INFECTIONS AMONG FEMALE SEX WORKERS IN KITUI}

\begin{abstract}
${ }^{1}$ Japheth Kioko*, ${ }^{2}$ Janet Musimbi, ${ }^{3}$ Parinita Bhattacharjee, ${ }^{4} J u d d i e$ Onyoni, ${ }^{5}$ Roselyne Nyakundi, ${ }^{6}$ Nicholas Ndone. ${ }^{1}$ Partners for Health and Development in Africa, Monitoring and Evaluation, Nairobi, Kenya; ${ }^{2}$ Partners for Health and Development in Africa, Outreach and Mobilization, Nairobi, Kenya; ${ }^{3}$ University of Manitoba, Centre for Global Public Health, Nairobi, Kenya; ${ }^{4}$ Partner for Health and Development in Africa, Outreach, Nairobi, Kenya; ${ }^{5}$ HopeWorldwide Kenya, Monitoring and Evaluation, Nairobi, Kenya; ${ }^{6}$ HopeWorldwide Kenya, Outreach, Embu, Kenya
\end{abstract}

\subsection{6/sextrans-2019-sti.772}

Background Genital infections such as Sexually Transmitted Infections (STI) increases the chances of acquiring and transmitting HIV. Kenya AIDS Indicator Survey showed that STI like syphilis was 2.5 times more common among people living with HIV. There is need to understand and respond to such co infections in a HIV prevention

Methods An analysis of cohort data of 1513 Female Sex Workers (FSW) enrolled and receiving HIV services in the KP clinic managed by Hope Word Wide, Kenya in Kitui County was conducted. Cohort data for the period of October 2017 to September 2018 was used and analysed for the STI and HIV screening and diagnosis. The analysis also segregated the analysis by age, above 24 years and below 24 years. Odds Ratio was calculated to measure the association.

Results Out of 1513 FSWs, 1391(92\%) received HIV testing services at least once in the year while 1448 (96\%) were screened for STI in the same period. A total of 130 (9\%) and 29 (2\%) FSWs received STI and HIV positive results respectively for the period. The Odds Ratio suggest that those FSWs who have STI had 1.2 times higher chances of being HIV positive. When desegregated by age, FSWs below 24 years with an STI had 2.1 times higher chances.

Conclusion There exists correlation between HIV and STI among Female Sex Workers in Kitui specially among those below 24 years. The intervention needs to screen, diagnose and treat STI among FSWs more aggressively as a response to HIV prevention.

Disclosure No significant relationships.

\section{P708 WOMEN'S ENCOUNTERS WITH VENUE-BASED HIV RISK CONTEXTS IN ABUJA, NIGERIA}

${ }^{1}$ Laura Thompson*, ${ }^{2}$ Kalada Green, ${ }^{2}$ Baba Mari, ${ }^{3}$ Shajy Isac, ${ }^{4}$ Ravi Prakash, ${ }^{2}$ Judith Ariri Edafe, ${ }^{2}$ Janet Halliday, ${ }^{5}$ Robert Lorway, ${ }^{6}$ James Blanchard. ${ }^{1}$ University of Manitoba, Centre for Global Public Health, Department of Community Health Sciences, Winnipeg, Canada ${ }^{2}$ Centre for Global Public Health - Nigeria, Abuja, Nigeria; ${ }^{3}$ India Health Action Trust, Bangalore, India; ${ }^{4}$ Karnataka Health Promotion Trust, Bangalore, India; ${ }^{5}$ Center for Global Public Health, Department of Community Health Sciences, University of Manitoba, Winnipeg, Canada; ${ }^{6}$ University of Manitoba, Centre for Global Public Health, Winnipeg, Canada

\subsection{6/sextrans-2019-sti.773}

Background Venues where people meet sexual partners are understood to be important locations where HIV transmission risk plays out and represent potential intervention points. Women involved in sex work and those seeking casual partners spend time in the same venues, forming sexual partnerships with some of the same people and experiencing the same risks. This study provides a characterization of key venues where women meet new sexual partners in Abuja, Nigeria, and describes the sexual behaviours, sexual networking patterns, and challenges experienced by women in these venues.

Methods Key informant interviews were used to characterize 836 venues where people congregate for social activities in Abuja, Nigeria, in terms of number of patrons, busy times, and availability of harm reduction supplies. A questionnaire capturing demographics, behaviours, health, and experiences of violence was administered to 892 women who participate in sex work or casual sex at a random sample of 105 of the profiled venues. Descriptive analysis was conducted with stratification by type of venue.

Results A diverse set of venues were identified, with bars/ nightclubs identified as having the highest volume of patrons. Most of the women indicated meeting partners at bars/nightclubs as well as hotels/lodges. Half of the women had experienced a miscarriage or abortion and perceived themselves to be at great risk of HIV infection. Eighteen percent had experienced condom breakage in the previous week, $15 \%$ had ever been arrested, and $8 \%$ had been beaten in the past year.

Conclusion A diverse set of women intermingle at different venues and have a diverse set of needs, including reproductive health, violence reduction, and infectious disease prevention. By re-orienting HIV programs towards venues where sexual partnerships form instead of towards specific key populations who are often blamed for transmission, the broader needs of 
a larger group of individuals who attend these venues may be addressed.

Disclosure No significant relationships.

\section{P709 ABNORMAL CERVICAL SCREENING TEST AND SEXUALLY TRANSMITTED INFECTIONS IN WEST AFRICA'S FEMALE SEX WORKERS}

${ }^{1}$ Ibrahima Téguété* ${ }^{2}$ ' $F$ atoumata Korika Tounkara, ${ }^{3}$ Fernand Guédou, ${ }^{4}$ Bintou Dembele, ${ }^{5}$ Michel Alary. ${ }^{1}$ Gabriel Toure Teaching Hospital, Bamako, Mali; ${ }^{2}$ Axe Santé des Populations et Pratiques Optimales en Santé, HSS, Social and Preventive Medicine, Québec, Canada; ${ }^{3}$ Dispensaire IST, Cotonou, Benin; ${ }^{4}$ ARCAD/SIDA, Bamako, Mali; ${ }^{5} \mathrm{CHU}$ de Quebec Université Laval, Quebec, Canada

\subsection{6/sextrans-2019-sti.774}

Background Cervical cancer is the most common cancer in women in Sub-Saharan Africa. The situation is worst among female sex workers (FSW), a population with poor access to quality reproductive health services. This study aimed to: (1) estimate the prevalence of abnormal cervical screening test (ACST) and (2) assess the association between ACST and sexually transmitted infections (STIs)/lower genital tract infections (LGTIs) among FSW in Cotonou (Benin) and Bamako (Mali).

Methods We conducted a cross-sectional study among nonpregnant FSW aged 18 to 64 years from April 2017 to February 2018. We used a peer recruitment sampling strategy in two West African cities. Visual inspection with acetic acid (5\%) and with Lugol's iodine (VIA/VIL) were performed to screen for cervical cancer. Women were also screened for STIs/LGTIs, specifically those with Trichomonas vaginalis (TV), Candida albicans (CA), Chlamydia trachomatis (CT), Neisseria gonorrhoeae (NG) and bacterial vaginosis (BV). ACST prevalence was computed and its association with STIs/LGTIs was assessed using multivariate logistic regression.

Results ACST prevalence was $20.2 \%$ among 312 FSW in Benin Vs. $10.5 \%$ among 353 FSW in Mali. Of these, 91.2\% never had cervical screening. Mean age at sexual debut was $16.3 \pm 3.0$ years. The overall STIs/LGTIs prevalence rates were: TV, 2.7\%; CT, 10.9\%; NG, 19.4\%; HIV, 23.0\%; CA, $7.4 \%$ and $\mathrm{BV}, 37.1 \%$. CA was the only infection associated with ACST $(\mathrm{aOR}=4.03$; 95\%CI: 1.77-9.17). Also, there was a statistical association between a coinfection by CA-TV and ACST $(\mathrm{aOR}=3.21 ; 95 \% \mathrm{CI}$ : 1.47-7.01). Finally, age at sexual debut $<10$ years old was significantly associated with ACST $(\mathrm{aOR}=6.10 ; 95 \% \mathrm{CI}: 1.19-31.21)$.

Conclusion The prevalence of ACST and STIs/LGTIs was very high; there is an obvious need to improve the diagnostic capability and the clinical management of these conditions among FSW of Sub-Saharan Africa.

Disclosure No significant relationships.

\section{P710 EPIDEMIOLOGY OF ABNORMAL CERVICAL CYTOLOGY IN FEMALE SEX WORKERS IN MALI, WEST AFRICA}

${ }^{1}$ Fatoumata Korika Tounkara*, ${ }^{2}$ Ibrahima Téguété, ${ }^{3}$ Fernand Guédou, ${ }^{4}$ Bintou Dembele, ${ }^{5}$ Michel Alary. 'Axe Santé des Populations et Pratiques Optimales en Santé, HSS, Social and Preventive Medicine, Québec, Canada; ${ }^{2}$ Gabriel Toure Teaching Hospital, Bamako, Mali; ${ }^{3}$ Dispensaire IST, Cotonou, Benin; ${ }^{4}$ ARCAD/SIDA, Bamako, Mali; ${ }^{5} \mathrm{CHU}$ de Quebec Université Laval, Quebec, Canada

10.1136/sextrans-2019-sti.775
Background To our knowledge, no previous study has been carried out about cervical cancer in female sex workers (FSW) in Mali. The objective of this work was to estimate the prevalence and risk factors of cervical precancerous lesions among FSW in Mali.

Methods We carried out a cross-sectional study from November 2017 to February 2018 in Mali. Screening for cervical cancer was performed with Papanicolaou test. HIV and syphilis serology, direct examination of vaginal smears for vaginitis and bacterial vaginosis and nucleic acid amplification tests for gonorrhea and chlamydia were also carried out. We calculated the prevalence rates. Risk factors of low grade squamous intraepithelial lesions (LSIL), and high grade squamous intraepithelial lesion (HSIL) were identified using polytomous logistic regression. Adjusted odd ratios (aOR) with 95\% confidence intervals $(95 \% \mathrm{CI})$ were estimated.

Results Among the 353 women enrolled in the study, 20.4\% were HIV seropositive, whereas $53.8 \%$ had at least one of other sexually transmitted infections (STIs), including trichomoniasis at 3.7\%; chlamydia, $14.0 \%$; gonorrhea, 24.4\%, syphilis, $3.1 \%$ and bacterial vaginosis (Nugent score $\geq 7$ ), $23.5 \%$. The mean age at first sexual intercourse was $15.3 \pm 2.9$ years. Fifty-eight women had abnormal cytology/histology (17.4\%; 95\% CI: $13.3-21.4 \%)$ and only $8.8 \%$ of FSW had at least one cervical screening test in the past year. Among all participants, $2.7 \%$ presented atypical glandular cells of undetermined significance (AGUS), $11.3 \%$ with LSIL, and 3.3\% with HSIL. Risk factors of LSIL were HIV infection $(\mathrm{aOR}=$ 1.53; 95\%CI: 1.02-2.30) and age at sexual debut $<10$ years $(\mathrm{aOR}=4.52 ; 95 \% \mathrm{CI}: 1.11-18.45)$. Factors associated with HSIL were self-reported previous STIs $(\mathrm{aOR}=4.92 ; 95 \% \mathrm{CI}$ : $1.15-21.09)$ and current syphilis $(\mathrm{aOR}=8.12$; 95\%CI: $1.86-$ 35.39).

Conclusion Low and high grades cervical squamous intraepithelial lesions have different risk factors profile in Malian FSWs.

Disclosure No significant relationships.

\section{P712 "YOU NEED A CASH BUFFER": MALE SEX WORK AND CONDOM USE IN THE ERA OF HIV PRE-EXPOSURE PROPHYLAXIS}

${ }^{1}$ Denton Callander*, ${ }^{2}$ Stephen Bell, ${ }^{3}$ Victor Minichiello, ${ }^{2}$ Ryan Deveau, ${ }^{2}$ Garrett Prestage, ${ }^{4}$ Basil Donovan. ${ }^{1}$ New York University, School of Medicine, New York, USA; ${ }^{2}$ UNSW Sydney, The Kirby Institute, Sydney, Australia; ${ }^{3}$ La Trobe University, Melbourne, Australia; ${ }^{4}$ UNSW Sydney, Kirby Institute, Sydney, Australia

\subsection{6/sextrans-2019-sti.776}

Background HIV pre-exposure prophylaxis (PrEP) has reshaped condom use practices in many contexts, but little is known about its impact on commercial sex encounters between men. This study investigated condom use in Australian male sex work.

Methods During 2017-2018, we undertook a mixed-method ethnographic study of male sex work in Australia, working with male sex workers $(n=32)$ and clients of sex work $(n=3)$ to collect online sex work profile data and conduct individual and group interviews. These data were analyzed topically with a focus on condom use.

Results In November 2018, online data were scraped from 236 male sex work profiles: $2.1 \%$ expressed an explicit requirement for condom use, down from 14.2\% in June 2017 